\title{
Electromagnetic Study of Multilayer Media
}

\author{
Joel P. Booth Stephanie Brown \\ U.S. Army Aviation and Missile Research, Development, \& Engineering Center \\ AMSRD-AMR-SG-RF \\ Redstone Arsenal, AL. 35898-5000 \\ (256) 876-2424 \\ Joel.booth@rdec.redstone.army.mil
}

\begin{abstract}
This paper will discuss a basic electromagnetic analysis of multilayer material used in a radome application. The purpose of this work is to investigate a possible new configuration for a missile seeker. The discussion will be based on computer simulations with real world applications. This effort is being conducted in the RF Technology Division of the Applied Sensors, Guidance, and Electronics Directorate in conjunction with End Game Analysis, collocated at the US Army Aviation and Missile Command, Aviation and Missile Research, Development, and Engineering Center (AMRDEC) on the Redstone Arsenal in Huntsville, Alabama. The ground work for this effort was covered in a graduate class at the University of Alabama in Huntsville under the instruction of Dr. John Jarem. This research is a continuation of that work.
\end{abstract}

The data obtained from this effort will be used in the selection of new missile seeker system parameters. The effects of the research could drive down cost manufacturing and create new design concepts. The results could also effect the combination of modes in seeker design used to achieve its mission.

\section{TABLE OF CONTENTS}

\section{BACKGROUND \\ II. Analysis \\ III. CONCLUSION \\ REFERENCES}

\section{BACKGROUND}

The purpose of this analysis is to investigate a concept for extending the range of beam steering in phased array missile seeker applications without the addition of a gimble or other means of mechanically positioning the array. Mechanical gimbal systems are large, costly and much slower than electronically steerable array systems. The ability to quickly steer a beam is a great advantage in military applications. It is possible to track several

\footnotetext{
${ }^{1}$ U.S. Government work not protected by U.S. copyright.

Paper number 1105 ver 2
}

incoming targets by quickly switching the beam between each target or through multiple beam generation. This can be achieved through electronically steerable arrays. In addition, the ability to overcome jamming through adaptive nulling is another advantage.

Electronically steered arrays are achieved by creating a phase shift between individual radiating elements usually with a time delay. By controlling the phase of each element, it is possible to direct the energy to a specific location, thereby steering the beam. Conventional beam steering is achieved by phase shifters, or phase elements that use switching mechanisms to achieve the necessary time delay between radiating elements. These phase shifters are therefore a digital system and true steering of every possible angle is not possible.

Digital phase shifters, or n-bit phase shifters, limit the angles to which any particular element may be steered. These angles are defined by phase shifter bits meaning that there are $2^{\mathrm{N}}$ possible phase divisions, where $\mathrm{N}$ is the number of bits. For example, if $\mathrm{N}=3$ then the shifter provides eight choices in phase division from input to output, and they are 0 (360), 45, 90, 135, 180, 225, 270, and 315 degrees. Each element can have a different phase added to its information. When all the elements are summed together in the array, beam steering is achieved [1]. Figure 1 shows a mathematical analysis of a 3-bit phase shifter used to steer a beam steered to $(10,35)$ degrees. The figure also shows the grating lobes that appear in the field of view of the array. 


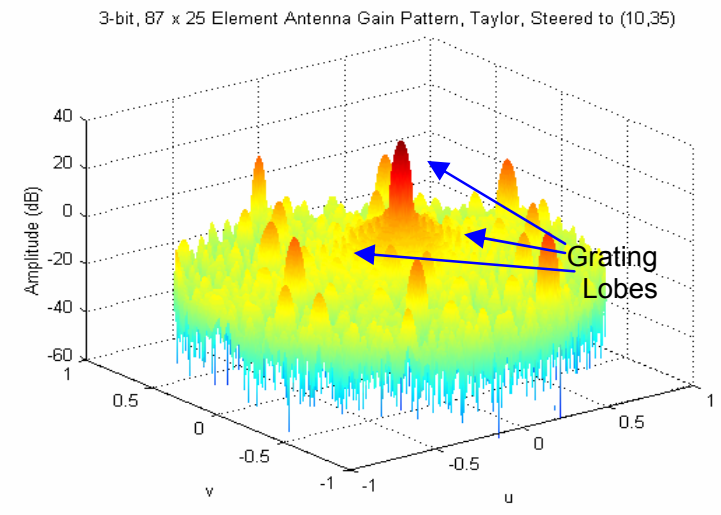

Figure 1. Beam Steered to (10,35) Degrees using a 3-bit Phase Shifter

Conventional limiting is important in that it prevents the appearance of grating lobes in the radiation pattern. The limiting is to prevent a return from a target being detected in out lobes of the array at the same time. However, there is a substantial loss in the range of view of the seeker due to this limiting. Current technology in phased array antenna systems has a limited range of beam steering. It is rare to find a phased array that is steerable more than sixty degrees off boresight. This problem is even more prevalent in digital systems where steering to specific angles is limited by the range of the phase shifter. Figure 2 gives a pictorial explanation of "degrees off boresight" beam steering.

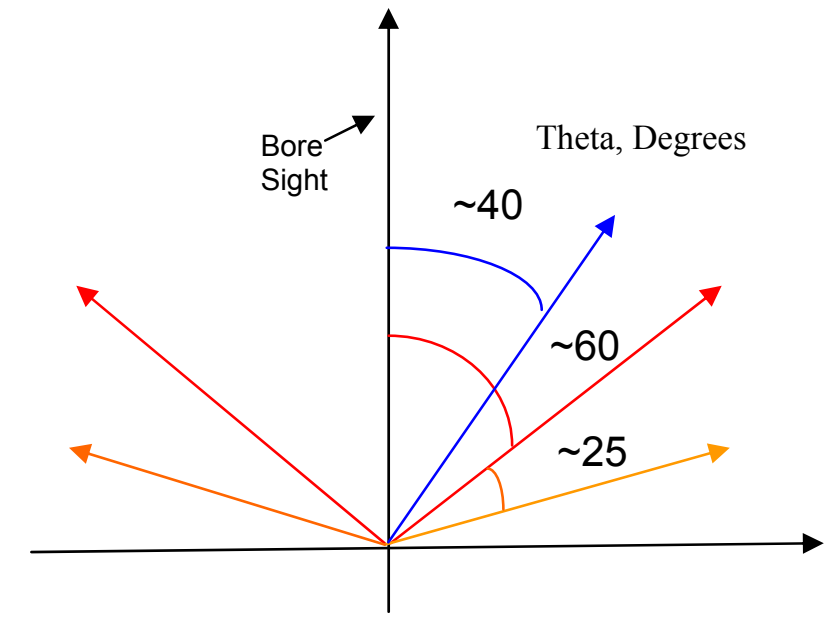

Figure 2. Pictorial Explanation of "Degrees off Boresight" Beam Steering.

One potential solution that allows for increasing the steering off boresight may be found in taking advantage of a material's natural tendency to bend fields depending on the nature of its dielectric properties. It is known that a material's index of refraction is related to its relative permittivity or dielectric constant as: [2]

$$
N=\sqrt{\varepsilon_{r}}
$$

A basic analysis of the behavior of electromagnetic fields in multilayer material can be used to explore the proposed method, of using multilayer materials to extend the steering range of a phased array while limiting grating lobes.

The first step in the analysis is to define Snell's law in terms of the problem. The physical set-up for the derivation of Snell's law is shown in Figure 3 below: [3]

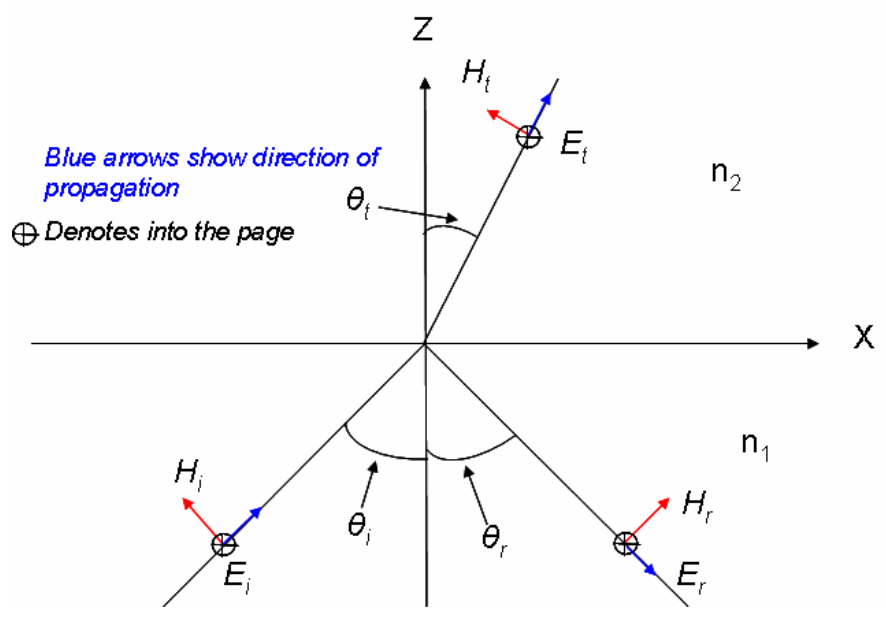

Figure 3. Pictorial Representation of Analysis

We will consider the incident field to be

$$
E_{y i}=E_{o} \exp \left(-j q_{i} z-j \beta_{i} x\right)
$$

Where $q_{i}=k_{l} \cos \theta_{i}, \beta_{i}=k_{l} \sin \theta_{i}$, and $k_{l}=\omega \sqrt{\mu_{1} \varepsilon_{1}}$ $=k_{o} n_{l} . k_{l}$ is the wave number of medium one and $k_{o}$ is the wave number of free space. The reflected field is given by

$$
E_{y r}=R_{s} E_{0} \exp \left(+j q_{r} z-j i \beta_{r} x\right)
$$

Where $R_{s}$ is the reflection coefficient and $q_{r}$ and $\beta_{r}$ are the same from substituting $\theta_{r}$ for $\theta_{i}$. The transmitted field is similarly represented by:

$$
E_{y t}=T_{s} E_{o} \exp \left(-j q_{t} z-j \beta_{t} x\right)
$$

Where $T_{s}$ is the transmission coefficient and $q_{t}$ and $\beta_{t}$ are represented by the substitution of $k_{2}$ and $\theta_{t}$ into the above equations. Now applying the boundary conditions at $z=0$, we get

$$
E_{y i}+E_{y r}=E_{y t}
$$

This leads us to the following relationship 
$\exp \left(-j \beta_{i} x\right)+R_{s} \exp \left(-j \beta_{r} x\right)=T_{s} \exp \left(-j \beta_{t} x\right)$

And for phase matching to hold for all $x$ we get

$$
\beta_{i}=\beta_{r}=\beta_{t}
$$

And this leads us to the following equation

$$
k_{i} \sin \theta_{i}=k_{r} \sin \theta_{r}=k_{t} \sin \theta_{t}
$$

From here by applying a few simple algebra steps we get Snell's law

$$
n_{1} \sin \theta_{i}=n_{2} \sin \theta_{t}
$$

The second step in the analysis is to apply Snell's law to multilayer media in the context of the problem. This analysis is done by simulating the field's behavior using Matlab. This analysis is a first look at the problem and a potential solution. The tool uses two major assumptions that should be noted. First, the electromagnetic wave is setup as a plane wave in the far field. Obviously, the region of interest is not in the far field in practice, but due to the frequency of interest, this is an acceptable analysis. A second assumption is that all of the materials are atomically smooth, meaning that there are no air gaps in the layers. While both of these assumptions would not stand up under great practical examination, they hold for this first level of analysis.

\section{ANALYSIS}

The Matlab code sets up a basic example of the manipulation of Snell's Law. The first example given is a simple boundary from air $\mathrm{n}=1$, through fiberglass $\mathrm{n}=$ 1.549. As expected, Figure 4 shows the ray is bending away from the boresight axis. The air to fiberglass boundary was chosen because of its practical relevance to the problem.

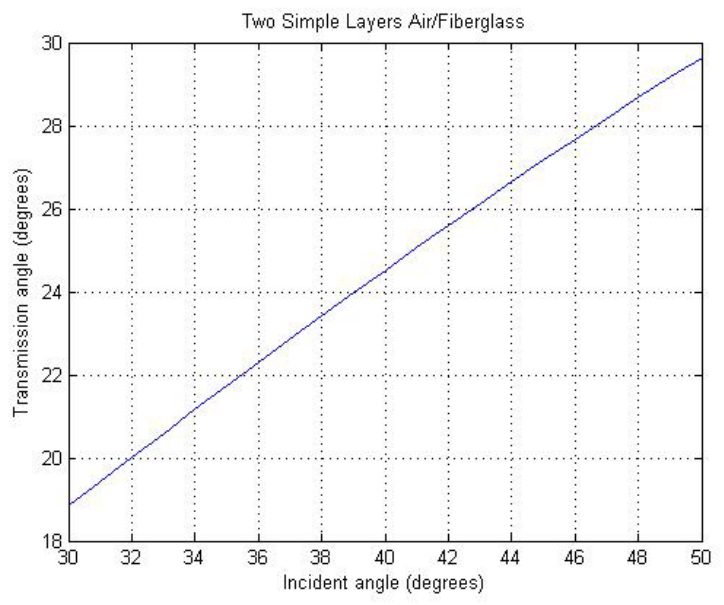

Figure 4. Air and Fiberglass Layers

However, looking at the second example (even closer to reality) of the air/fiberglass/air boundaries the effect of Snell's law is seen. Because the first and final layers are composed of the same dielectric, i.e. air, the incident angles are equal to the transmission angles. This effect is shown in Figure 5. hence the first difficulty in using typical ray bending methods to solve the phased-array problem.

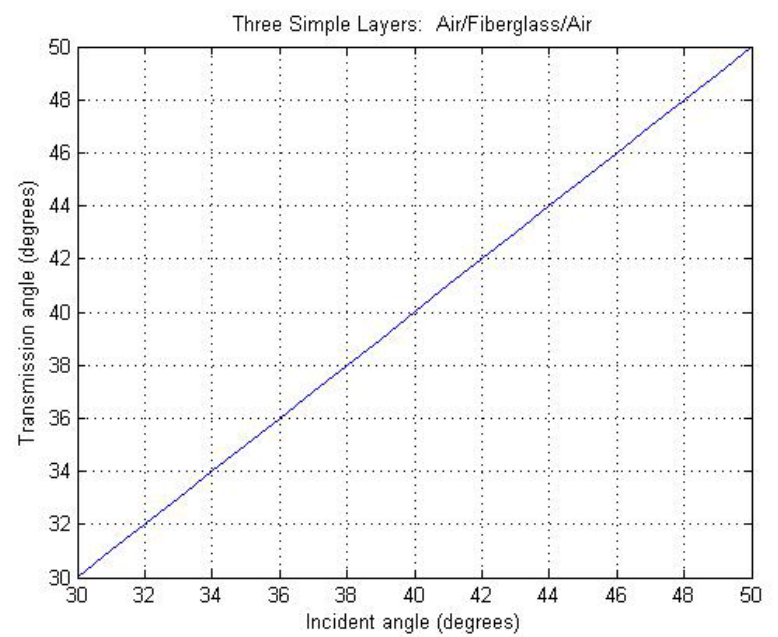

Figure 5. Air/Fiberglass/Air Layers

Now we will look at an example where the electromagnetic wave is incident in a material other than air. As demonstrated in example 3 of the code and the resultant Figure 6, changing the material in which the ray is incident will allow for the desired ray bending. Figures 6 shows the dielectric constant varying as 2.1025, 2.1904, 2.298, and 2.4. These are the values obtained for different oils.[4] It would not be practical to use oil in a missile seeker, however this was a common material that is readily available and may be used to demonstrate the theory. Also note that the code uses the index of refraction of these materials which is found using the dielectric constants. The figure titles reflect the index of reflection. As the dielectric 
constant is increased, the bending angle increases.

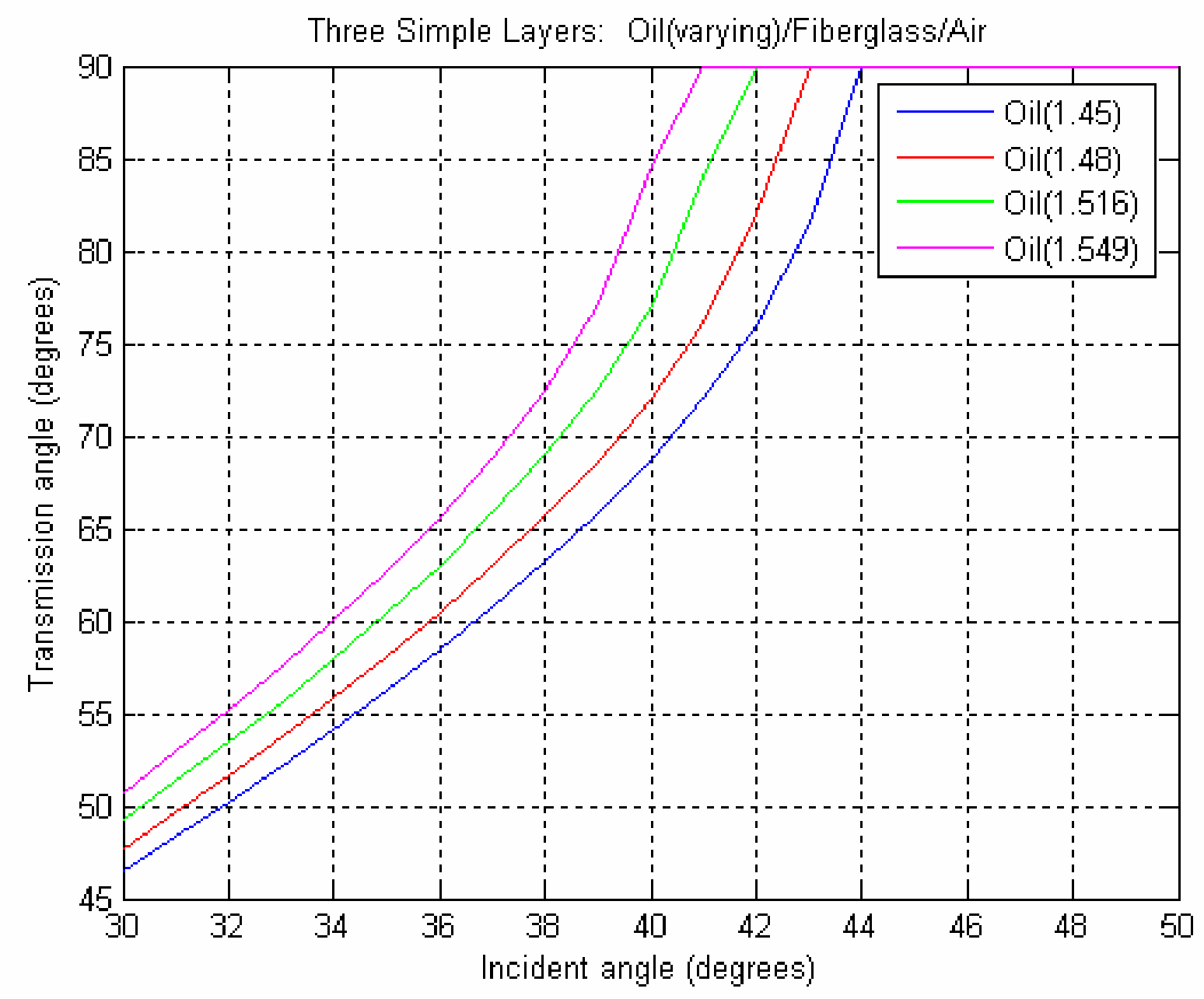

Figure 6. Varying Oil Index of Refraction

Figure 7 show illustrates the case when energy is beginning in air and returning to air. It is easily seen that is does not matter how many layers it passes through it will always leave at the same angle as it entered.

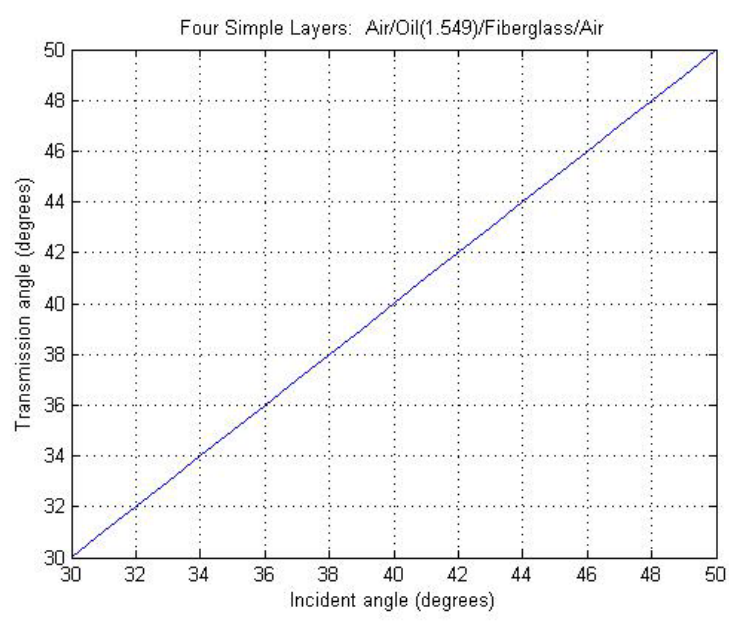

Figure 7. Air/Oil (1.549)/Fiberglass/Air Layers 


\section{CONCLUSION}

In the process of this analysis it was found that if the electromagnetic energy begins and ends in the same material it will always propagate in the same direction as it was before entering the bending material. This is a physical constant that is not obvious from Snell's law. Assuming all layers are atomically bonded such that no air gap is present between the layers and that near field effects are negligible, it is possible to alter the direction of propagation of electromagnetic energy. This will only hold if the beginning material and ending material are different, meaning that for propagation in air the electromagnetic energy must be launched in a different material initially. It has been shown that as the index of refraction increases the angle of bending also increases. This property could be used to increase the range of steering off of boresight.

Since it is not practical to launch the electromagnetic wave directly into a material such as oil having a higher index of refraction than air, other methods must be investigated. The follow-on research will involve anisotropic materials with permittivity tensors to change the propagation velocity of the wave. In addition, using a layering method where a grating is created throughout the radome material will be studied. Figure 8 gives a 2-D pictorial representation of this study.

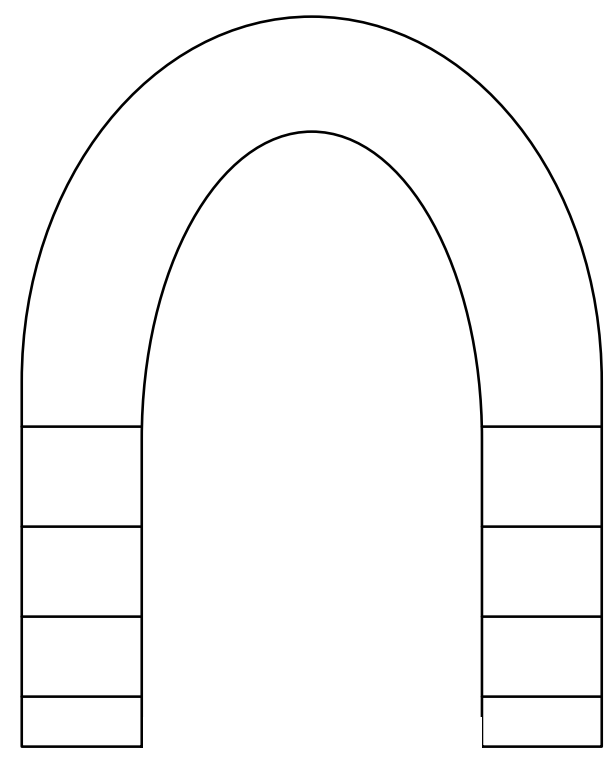

Figure 8. 2-D Pictorial Representation of Grating Study

Here, the lower materials of the radome are layered to bend the electromagnetic energy away from boresight once the beam enters these regions of the radome material. Electromagnetic wave propagation using Bragg gratings will be studied with anisotropic materials to determine the most efficient means of achieving the desired results.
Additional analysis to determine the reflection and transmission coefficients of the wave will be done to determine the overall efficiency of the radome. In missile applications, high performance is necessary and radome losses must be kept at a minimum. Multi-path wave propagation due to the gratings created to help bend the wave will need to be studied and their interference minimized.

Several different theoretical analysis are expected to be conducted before an actual radome is constructed. The results of these analysis will be used to determine the material properties necessary for the final design.

\section{REFERENCES:}

[1] " A Mathematical Comparison of Ideal Versus N-bit Phase Shifters (U)", September 2003, Janice Rock U.S. Army Aviation and Missile Command

[2] Balanis, Constantine A.," Advanced Engineering Electromagnetics", John Wiley and Sons 1989.

[3] Ishimaru, Akira ,'Electromagnetic Wave Propagation, Radiation, and Scattering," Prentice Hall 1991.

[4] "Dielectric Constants Chart", http://www.asiinstr.com/technical/Dielectric\%20Constants.ht $\underline{\mathrm{m}}$, accessed 10/13/2005 


\section{BIOGRAPHY}

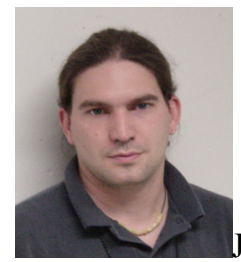

Joel P. Booth is a research engineer with the Applied Sensors, Guidance, and Electronics Directorate of the U.S. Army Aviation and Missile Research Development Engineering coman; Redstone Arsenal. He graduated with a B.S. in Electrical engineering from the University of Alabama in Huntsville.

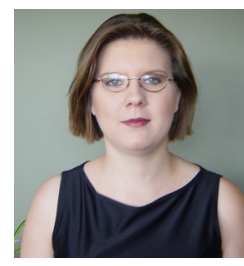

Stephanie E. Brown is a research engineer with the System Simulation and Development Directorate of the U.S. Army Aviation and Missile Research, Development Engineering Command; Redstone Arsenal. She graduated with a B.S. in Electrical Engineering from the University of Alabama in Huntsville. 